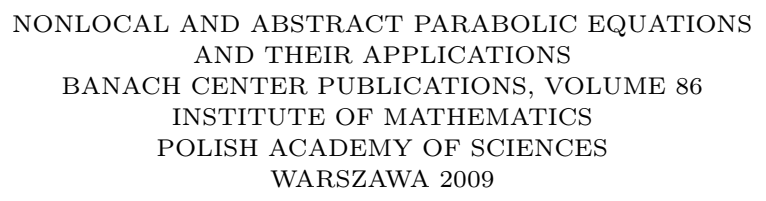

\title{
ON RADIALLY SYMMETRIC SOLUTIONS OF SOME CHEMOTAXIS SYSTEM
}

\author{
ROBERT STAŃCZY \\ Instytut Matematyczny, Uniwersytet Wrockawski \\ pl. Grunwaldzki 2/4, 50-384 Wrocław, Poland \\ E-mail: stanczr@math.uni.wroc.pl
}

\begin{abstract}
This paper contains some results concerning self-similar radial solutions for some system of chemotaxis. This kind of solutions describe asymptotic profiles of arbitrary solutions with small mass. Our approach is based on a fixed point analysis for an appropriate integral operator acting on a suitably defined convex subset of some cone in the space of bounded and continuous functions.
\end{abstract}

1. Introduction. In this paper we study nonlocal, singular boundary value problem

$$
\begin{array}{r}
\Phi^{\prime \prime}(y)+\frac{1}{4} \Phi^{\prime}(y)+\frac{1}{2 y} \Phi^{\prime}(y) e^{-\tau y / 4}\left(\int_{0}^{y} e^{\tau z / 4} \Phi^{\prime}(z) d z\right)=0 \\
\Phi(0)=0, \quad \Phi(\infty)=\frac{M}{2 \pi},
\end{array}
$$

arising from the following parabolic system modelling chemotaxis

$$
\begin{aligned}
\frac{\partial u}{\partial t} & =\nabla \cdot(\nabla u-u \nabla v), \quad x \in \mathbb{R}^{2}, t>0, \\
\tau \frac{\partial v}{\partial t} & =\Delta v+u, \quad x \in \mathbb{R}^{2}, t>0 .
\end{aligned}
$$

as a self-similar profiles considered e.g. by Yüki Naito in [8] with $\tau=1$. The author proves therein that for $\tau=1$ and total mass $0 \leq M=\int_{\mathbb{R}^{2}} u$ small enough the solutions behave asymptotically like self-similar solutions of that system. More specifically, by self-similar

2000 Mathematics Subject Classification: 35Q80, 35K60, 35B40, 45G10, 47H10.

Key words and phrases: self-similar solutions, chemotaxis system, nonlocal problems.

The paper is in final form and no version of it will be published elsewhere. 
solutions of (2)-(3) we mean the solutions obtained by the change of variables

$$
\begin{array}{r}
u=\frac{1}{t} W\left(\frac{|x|}{\sqrt{t}}\right), \quad v=Z\left(\frac{|x|}{\sqrt{t}}\right), \\
\Phi(y)=\frac{1}{2} \int_{0}^{y} W(\sqrt{t}) d t, \quad \Psi(y)=\frac{1}{2} \int_{0}^{y} Z(\sqrt{t}) d t,
\end{array}
$$

which allows us to reduce the problem (2)-(3) to (1). These special solutions can play an important role as asymptotic self-similar profiles for the original problem (2)-(3) as was stressed on before.

In this paper we shall consider a more general system with the parameter $\tau>0$ as in [4], [10] or in a survey article [9]. The case of the parabolic-elliptic system with $\tau=0$ has been considered in [3] and [5].

The authors of [5] proved asymptotically self-similar behavior of solutions of (2)-(3) with $\tau=0$ using entropy methods. It was proved in [1], [3] that given $0 \leq M<8 \pi$ self-similar solutions exist and they are unique. For $M>8 \pi$ there are no solutions of (1).

The related models have been considered e.g. in [6], [12] and [13].

For the parabolic system (2)-(3) the determination of the optimal range of $M$ leading to the existence of self-similar solutions for a given $\tau$ and their uniqueness with a given $M$ seem to be an open problem. They are determined by the equation (1). The results in this direction can be found in [10], where preliminary estimates from [2] for $\tau=1$ had been greatly improved. In particular, the authors of [10] proved that

- if $0<\tau \leq 1 / 2$, then solutions of (1) exist exactly for $M \in[0,8 \pi)$,

- if $\tau \in(1 / 2,1]$, then a necessary condition for the existence of solutions of (1) reads $M<4 \pi^{3} / 3$,

- if $\tau>1$, then a necessary condition for the existence of solutions of (1) reads $M<\tau^{2} 4 \pi^{3} / 3$.

Then, the last result was improved in [4] to

$\dagger$ if $\tau>1$, then a necessary condition for the existence of solutions of (1) reads $\frac{M}{2 \pi}<\min \left\{\tau \frac{2}{3} \pi^{2}, 4(\tau+1)\right\}$.

The last three properties provide only a priori bounds for the parameters and thus the existence in the full range of parameters is not guaranteed. As for the existence result it was shown in [10, Th. 2] that self-similar solutions form a one-parameter family parametrized by $\tau>0$, and their $L^{1}$ norms tend to $8 \pi$ as the parameter goes to $\infty$.

Thus, the uniqueness does not hold if there is a self-similar solution for $M>8 \pi$.

It should be noted that the solutions of (1) are smooth as a consequence of an analysis of the self-similar solutions of the original system (2)-(3) without radial symmetry assumptions. In fact, each self-similar solution of (2)-(3) is radial, [10, Th. 3]. A different construction of self-similar solutions of (2)-(3) was given in [2].

The results in [8, Th. 3] allowed the author to obtain the uniqueness in a certain class of functions of sufficiently small self-similar solutions. The proof involved the use of perturbation arguments via the contraction mapping theorem. 
The problem of the uniqueness of self-similar solutions mentioned in [8, Remark 1 (i)] is important. If this was true it would permit one to prove a stronger version of Theorem 3 in [8], i.e. the asymptotics of any global in time solution $u$ is described by that of the unique self-similar solution corresponding to the same mass $M$ as that for $u$.

Whether this property holds for (1) is an open question as was stated in [4]. Some numerical experiments carried out by J. Dolbeault for $\tau>0.9$ suggest that it is not the case. Moreover, it should be noted that for some nonlinear parabolic equations there are multiple self-similar solutions, see e.g. [7].

In view of the previous considerations, the uniqueness would not hold if there was a self-similar solution for $M>8 \pi$.

The problem (1) with the boundary conditions can be reduced to some integral equation. Indeed, it can be transformed into

$$
\left(\Phi^{\prime}(y) e^{y / 4}\right)^{\prime}+\frac{1}{2} e^{y / 4} \mathcal{T} \Phi^{\prime}(y)=0
$$

where the operator $\mathcal{T}$ is defined by

$$
\mathcal{T} \phi(y)=\frac{1}{y} \phi(y) e^{-\tau y / 4} \int_{0}^{y} \phi(z) e^{\tau z / 4} d z .
$$

Integrating (4) and multiplying by $e^{-y / 4}$ one obtains

$$
\Phi^{\prime}(y)=\Phi^{\prime}(0) e^{-y / 4}-\frac{1}{2} \mathcal{S} \mathcal{T} \Phi^{\prime}(y),
$$

where the linear operator $\mathcal{S}$ is defined as

$$
\mathcal{S} \phi(y)=e^{-y / 4} \int_{0}^{y} \phi(z) e^{z / 4} d z .
$$

Next, integrating (6) once again from 0 to $\infty$ and using mass constraint $\int_{0}^{\infty} \Phi^{\prime}(y) d y=\frac{M}{2 \pi}$ one can plug the value of $\Phi^{\prime}(0)$ in (6) to get the following equation

$$
\Phi^{\prime}(y)=\left(\frac{M}{8 \pi}+\frac{1}{8} \int_{0}^{\infty} \mathcal{S} \mathcal{T} \Phi^{\prime}(y) d y\right) e^{-y / 4}-\frac{1}{2} \mathcal{S} \mathcal{T} \Phi^{\prime}(y) .
$$

Thus setting $\phi=\Phi^{\prime}$ and defining the operator $\mathcal{P}$ by

$$
\mathcal{P} \phi(y)=\left(\frac{M}{8 \pi}+\frac{1}{8} \int_{0}^{\infty} \mathcal{S} \mathcal{T} \phi(y) d y\right) e^{-y / 4}-\frac{1}{2} \mathcal{S} \mathcal{T} \phi(y)
$$

the problem (1) can be reduced to looking for a fixed point of the operator $\mathcal{P}$.

We announce the main result to be proven in the next section.

THEOREM 1.1. If the mass parameter $M$ is small enough then the operator $\mathcal{P}$ has a fixed point and therefore (1) has a solution.

This theorem gives an alternative approach to the existence of self-similar solutions problem, cf. [2] and [10], where for each $\tau>0$ and $M \in(0,8 \pi)$ the existence of such solutions has been shown. We also show that the positivity property requires in our approach an assumption on $M<8 \pi$, cf. Lemma 2.1 . Finally we prove in Lemma 2.2 that solutions concentrated at 0 are not allowed. 


\section{Notation.}

- We denote by $B C([0, \infty))$ the space of bounded and continuous functions defined on the interval $[0, \infty)$ equipped with the sup norm $|\phi|_{\infty}=\sup _{y \geq 0}|\phi(y)|$.

- $B_{R}=B(0, R)$ denotes the closed ball in the space $B C([0, \infty))$.

- By the arrow $\searrow$ we denote the monotonicity property of a nonincreasing function.

- We set $m=\frac{M}{2 \pi}$.

2. Main results. First, we will state some properties of the operator $\mathcal{P}$ and the operators $\mathcal{T}, \mathcal{S}$ appearing in the definition of $\mathcal{P}$. Namely, setting

$$
m=\frac{M}{2 \pi},
$$

we can list the following statements.

(A) $\int_{0}^{\infty} \mathcal{P} \phi(z) d z=m$ for any $\tau>0$ if only all the terms in (9) are well defined.

(B) $\mathcal{P} \phi(y) e^{y / 4} \searrow$, i.e. the function is nonincreasing, for any $\phi \geq 0$ and any $\tau>0$.

(C) For any positive $\phi$ such that $\phi(y) e^{y / 4} \searrow$ we have

$$
\begin{array}{ll}
\mathcal{T} \phi(y) \geq \phi(y)^{2} e^{(1-\tau) y / 4} & \text { for any } \tau \geq 1, \\
\mathcal{T} \phi(y) \geq \phi(y)^{2} & \text { for any } \tau \leq 1 .
\end{array}
$$

For $\tau>1$ a more accurate estimate is available, i.e.

$$
\mathcal{T} \phi(y) \geq \frac{4}{y(\tau-1)} \phi(y)^{2}\left(1-e^{(1-\tau) y / 4}\right) .
$$

(D) For any positive $\phi$ such that $\phi(y) e^{y / 4} \searrow$ we have

$$
\mathcal{S T} \phi(y) \geq \phi(y) \int_{0}^{y} \phi(z) e^{(1-\tau) z / 4} d z \quad \text { for any } \tau \geq 1,
$$

while

$$
\mathcal{S} \mathcal{T} \phi(y) \geq \phi(y) \int_{0}^{y} \phi(z) d z \quad \text { and } \quad \int_{0}^{\infty} \mathcal{S} \mathcal{T} \phi(y) d y \geq \frac{1}{2} m^{2}
$$

for $\int_{0}^{\infty} \phi(z) d z=m$ and any $\tau \leq 1$.

(E) For any $\tau>0$ and any $\phi$ such that $\phi(y) e^{y / 4} \searrow$ and $\int_{0}^{\infty} \phi(z) d z=m$ we have the estimates

$$
\mathcal{T} \phi(y) \leq|\phi|_{\infty} \phi(y) e^{-\min \{\tau, 1\} y / 4} \quad \text { and } \quad \mathcal{T} \phi(y) \leq \frac{m}{y} \phi(y)
$$

(F) We have

$$
\begin{array}{ll}
\mathcal{T} \phi(y) \leq|\phi|_{\infty}^{2} e^{-(\tau+1) y / 4} & \text { if } \phi(y) e^{1 / 4 y} \searrow \text { and } \tau \leq 1 \\
\mathcal{T} \phi(y) \leq|\phi|_{\infty}^{2} e^{-y / 2} & \text { if } \phi(y) e^{1 / 4 y} \searrow \text { and } \tau \geq 1 .
\end{array}
$$

(G) $\mathcal{S T} \phi(y) \leq|\phi|_{\infty} m e^{-y / 4}$ if $\int_{0}^{\infty} \phi(y) d y=m$ and $\phi(y) e^{1 / 4 y} \searrow$.

(H) We can estimate

$$
\mathcal{S} \mathcal{T} \phi(y) \leq|\phi|_{\infty}^{2} \frac{4}{\tau}\left(e^{-y / 4}-e^{-(\tau+1) y / 4}\right) \quad \text { if } \phi(y) e^{1 / 4 y} \searrow \text { and } \tau \leq 1
$$


while

$$
\mathcal{S T} \phi(y) \leq|\phi|_{\infty}^{2} 4\left(e^{-y / 4}-e^{-y / 2}\right) \quad \text { for } \phi(y) e^{1 / 4 y} \searrow \text { and } \tau \geq 1
$$

(I) For any $\tau \leq 1$ and $\phi$ such that $\int_{0}^{\infty} \phi(y) d y=m$ and $\phi(y) e^{1 / 4 y} \searrow$ we have the following lower bounds

$$
\sup _{y \geq 0} \phi(y) e^{y / 4} \geq m / 4 \quad \text { and } \quad \sup _{y \geq 0} \mathcal{P} \phi(y) e^{y / 4} \geq m^{2} / 16+m / 4 .
$$

(J) $\mathcal{P}\left(\frac{1}{4} m e^{-y / 4}\right)=\frac{1}{2} m e^{-y / 2}$ for any positive $m=\frac{M}{2 \pi}$ and $\tau=1$.

We relegate the proofs of the above claims to Appendix. Now we find some convex invariant set under the action of the compact operator $\mathcal{P}$.

Lemma 2.1. The operator $\mathcal{P}$ is compact and maps the set $K \cap B(0, R)$ into $K$ where

$$
K=\left\{\phi \in B C([0, \infty)): \phi(y) \geq 0, \int_{0}^{\infty} \phi(z) d z=\frac{M}{2 \pi} \text { and } \phi(y) e^{1 / 4 y} \searrow\right\},
$$

$M<8 \pi$ and $R^{2} \leq \frac{M}{8 \pi}$ for $\tau>1$ or $R^{2} \leq \frac{\tau}{2}\left(\frac{M}{8 \pi}+\frac{M^{2}}{64 \pi}\right)$ for $\tau \leq 1$.

Proof. First of all the operators $\mathcal{T}$ and $\mathcal{S}$, by (E) and (G), are both bounded and positive, i.e. mapping positive functions into positive ones. The proof of the compactness of the operator $\mathcal{P}$ is a standard application of the Ascoli-Arzelà theorem if we use the asymptotic decay property of the operator $\mathcal{P}$ i.e. $\mathcal{P} \phi(y) \leq \mathcal{P} \phi(0) e^{-y / 4}$ following directly from the property (B) together with the estimate $\mathcal{P} \phi(y) \geq-\frac{1}{2} \mathcal{S} \mathcal{T} \phi(y)$ and estimate $(\mathrm{G})$. We use here the boundedness of both $\mathcal{T}$ and $\mathcal{S}$. For the compactness criterion in the space of bounded and continuous functions one can see, e.g., [11].

The property of the invariance of the part of the set $K$ related to $B(0, R)$ under the action of the operator $\mathcal{P}$ follows from straightforward calculations to be presented below. Due to the property (B) the only nontrivial fact which guarantees that $\mathcal{P}: K \cap B(0, R) \rightarrow$ $K$ is that $\mathcal{P} \phi(y) \geq 0$. This inequality, by definition of $\mathcal{P}(9)$ and property (B), is equivalent to

$$
\frac{m}{4}+\frac{1}{8} \int_{0}^{\infty} \mathcal{S} \mathcal{T} \phi(y) d y-\frac{1}{2} \lim _{y \rightarrow \infty} \mathcal{S} \mathcal{T} \phi(y) e^{y / 4} \geq 0
$$

This condition can be rephrased as

$$
\frac{m}{4}+\frac{1}{8} \int_{0}^{\infty} \mathcal{S} \mathcal{T} \phi(y) d y-\frac{1}{2} \int_{0}^{\infty} \mathcal{T} \phi(y) e^{y / 4} d y \geq 0 .
$$

This is guaranteed by the relation $\int_{0}^{\infty} \mathcal{S} \mathcal{T} \phi(y) d y=4 \int_{0}^{\infty} \mathcal{T} \phi(y) d y$ and the second property from $(\mathrm{F})$, i.e. $\mathcal{T} \phi(y) \leq|\phi|_{\infty}^{2} e^{-y / 2}$ for any $\tau \geq 1$, as

$$
\frac{1}{2} \int_{0}^{\infty} \mathcal{T} \phi(y)\left(e^{y / 4}-1\right) d y \leq R^{2}
$$

and

$$
R^{2} \leq \frac{m}{4}
$$


For $\tau \leq 1$ to guarantee (12) we use (D) $\int_{0}^{\infty} \mathcal{S} \mathcal{T} \phi(y) d y \geq m^{2} / 2$ and (E) $\mathcal{T} \phi(y) \leq$ $R \phi(y) e^{-\tau y / 4}$ which implies

or

$$
\frac{m}{4}+\frac{m^{2}}{16}-\frac{R}{2} \int_{0}^{\infty} \phi(y) e^{(1-\tau) y / 4} d y \geq \frac{m}{4}+\frac{m^{2}}{16}-\frac{2}{\tau} R^{2} \geq 0
$$

$$
R^{2} \leq \frac{\tau}{2}\left(\frac{m}{4}+\frac{m^{2}}{16}\right)
$$

On the other hand by the property $(\mathrm{B}), m=\int_{0}^{\infty} \phi(y) d y \leq 4 R$. Combining this estimate with either (13) or (14) we can see that necessarily $m \leq 4$ and $M \leq 8 \pi$, which seems to be a natural condition for our considerations.

LEMma 2.2. For $1<\tau<2$ there are no fixed points of the integral operator (9) such that

$$
\int_{0}^{\infty} y \phi(y)^{2} d y>2 \int_{0}^{\infty} \phi(y) d y
$$

and thus no solutions to equation (1) exist. More generally, for arbitrary $\tau>1$ a similar condition guaranteeing nonexistence can be stated

$$
\int_{0}^{\infty} \psi(y) \phi(y)^{2} d y>\int_{0}^{\infty} \phi(y) d y
$$

where the function $\psi$ is defined by

$$
\psi(y)=\frac{8}{y(\tau-1)}\left(1-e^{(1-\tau) y / 4}\right)\left(e^{y / 4}-1\right) .
$$

Proof. Let us recall that by integration by parts we have $\int_{0}^{\infty} \mathcal{S} \mathcal{T} \phi(y) d y=4 \int_{0}^{\infty} \mathcal{T} \phi(y) d y$, thus positivity of $\mathcal{P} \phi$ (11) rephrased as (12) is violated if the limit of the function $\mathcal{P} \phi(y) e^{y / 4}$ at $\infty$ is negative, i.e.

$$
\frac{m}{4}+\frac{1}{2} \int_{0}^{\infty} \mathcal{T} \phi(y)\left(1-e^{y / 4}\right) d y<0
$$

or

$$
\int_{0}^{\infty} \mathcal{T} \phi(y)\left(e^{y / 4}-1\right) d y>\frac{m}{2}
$$

Next, by the first property from (C) the inequality (18) follows from

$$
\int_{0}^{\infty} \chi(y) \phi(y)^{2} d y>\frac{m}{2},
$$

where the function $\chi$ is defined by $\chi(y)=e^{(2-\tau) y / 4}-e^{(1-\tau) y / 4}$. Then expanding the function $\chi$ in Taylor series and neglecting positive terms but the first one (the zero order terms cancel out) one can use for $1<\tau<2$ the estimate $\chi(y) \geq y / 4$ to estimate (19) and get $\frac{1}{4} \int_{0}^{\infty} y \phi(y)^{2} d y>\frac{m}{2}$ whence the announced estimate follows.

To show (16) we use essentially the same estimate as before, i.e. (18), but this time we use the last estimate from $(\mathrm{C})$ to get the claim.

Now we state the invariance of some convex set under the action of $\mathcal{P}$.

THEOREM 2.3. If the mass parameter $m$ is small enough then the operator $\mathcal{P}$ maps some ball $B_{R}$ intersected with the convex set $K$ in the space of bounded and continuous functions 
equipped with the sup norm into $B_{R}$. To be more specific we require for $m<1$ that $\tau>1$ or $\frac{8 m}{(4+m)(2-m)^{2}}<\tau \leq 1$.

Proof. First of all the operators $\mathcal{T}$ and $\mathcal{S}$, by (E) and (G), are both bounded and positive, i.e. mapping positive functions into positive ones. Next note that, due to the monotonicity property $\mathcal{P} \phi(y) e^{1 / 4 y} \searrow$ stated in $(\mathrm{B})$, the sup norm of $\mathcal{P} \phi$ is attained at 0 and since $\mathcal{S T} \phi(0)=0$ it can be estimated, for any $|\phi|_{\infty} \leq R$, as follows

$$
\begin{aligned}
|\mathcal{P} \phi|_{\infty} & =\mathcal{P} \phi(0)=\frac{M}{8 \pi}+\frac{1}{8} \int_{0}^{\infty} \mathcal{S} \mathcal{T} \phi(y) d y=\frac{M}{8 \pi}+\frac{1}{2} \int_{0}^{\infty} \mathcal{T} \phi(y) d y \\
& \leq \frac{M}{8 \pi}+\frac{M}{4 \pi}|\phi|_{\infty} \leq \frac{M}{8 \pi}+\frac{M}{4 \pi} R .
\end{aligned}
$$

In the above estimate we integrated by parts to get $\int_{0}^{\infty} \mathcal{S} \mathcal{T} \phi(y) d y=4 \int_{0}^{\infty} \mathcal{T} \phi(y) d y$ and used the estimate $\mathcal{T} \phi(y) \leq|\phi|_{\infty} \phi(y)$ following directly from (E). Next we use Lemma 2.1. Recall that the convex set

$$
B(0, R) \cap\left\{\phi \in B C: \phi(y) \geq 0, \int_{0}^{\infty} \phi(z) d z=\frac{M}{2 \pi}, \phi(y) e^{1 / 4 y} \searrow\right\}
$$

is mapped by the operator $\mathcal{P}$ into $K$. Moreover, if $R$ is large enough to guarantee

$$
\frac{M}{8 \pi}+\frac{M}{4 \pi} R \leq R
$$

which is satisfied if the coefficient $M$ is less than $4 \pi$, then $\mathcal{P}$ maps $B(0, R) \cap K$ into $B(0, R)$.

Thus to guarantee the invariance of the set $B(0, R) \cap K$ under the action of the operator $\mathcal{P}$ for $\tau>1$ we have to combine (20) expressed equivalently for $m=\frac{M}{2 \pi}<2$ as

$$
R \geq \frac{m / 4}{1-m / 2}
$$

with condition (13). Hence we end up with the following condition $m^{2}-5 m+4>0$. This holds in view of $m<2$ for any $m<1$.

For $\tau \leq 1$ (21) and (14) yield

$$
\left(\frac{m / 4}{1-m / 2}\right)^{2}<\frac{\tau}{2}\left(\frac{m}{4}+\frac{m^{2}}{16}\right)
$$

whence

$$
\tau>\frac{8 m}{(4+m)(2-m)^{2}}
$$

The compactness property of $\mathcal{P}$ following from Lemma 2.1 together with invariance of the set $K \cap B(0, R)$ under the action of the operator $\mathcal{P}$ guaranteed by Theorem 2.3 suffice to use the Schauder theorem and obtain a fixed point for the operator $\mathcal{P}$. Thus we have proved the main Theorem 1.1 stated in the introduction.

Thus we have obtained a fixed point of $\mathcal{P}$ and a solution to (1) or self-similar one to the corresponding parabolic system.

REMARK 2.4. Although the range of the parameter is not optimal compared to the previously known results, it should be noted that the invariance of the convex set $K$, guaranteeing the positivity property of the solutions, appears in this approach to be of 
more importance as it naturally yields the necessary estimate $M \leq 8 \pi$. It suggests that the probable lack of an existence result for $M>8 \pi$ can be attributed to the requirement of the nonnegativity of the solutions both for the stationary and evolutionary problems. We hope that this might shed a new light on the problem and help to provide more thorough understanding of the criticality of the $8 \pi$ value of the mass parameter.

3. Appendix. Justifications of the claims $(\mathrm{A})-(\mathrm{J})$ from the previous section are given below.

(A) This property follows immediately from (9) by integration since the last two terms cancel out.

(B) This property follows from definitions (5), (7) and (9) if we multiply $\mathcal{P} \phi(y)$ by $e^{y / 4}$, get $-\frac{1}{2} \int_{0}^{y} \mathcal{T} \phi(y) e^{y / 4} d y+$ const and use the nonnegativity of $\mathcal{T} \phi$ implied by $\phi \geq 0$.

(C) We estimate using (5) and monotonicity of the function $\phi(y) e^{y / 4} \searrow$ as follows

$$
\mathcal{T} \phi(y)=\frac{1}{y} \phi(y) e^{-\tau y / 4} \int_{0}^{y} \phi(z) e^{\tau z / 4} d z \geq \frac{1}{y} \phi(y) e^{-\tau y / 4} \int_{0}^{y} \phi(y) e^{y / 4} e^{(\tau-1) z / 4} d z .
$$

Next, for $\tau \geq 1$, we drop the term $e^{(\tau-1) z / 4}$ estimating it from below by 1 . For $\tau \leq 1$ since the function $\phi(z) e^{\tau z / 4}$ is nonincreasing therefore $\phi(z) e^{\tau z / 4} \geq \phi(y) e^{\tau y / 4}$ for any $z \leq y$ thus

$$
\mathcal{T} \phi(y) \geq \frac{1}{y} \phi(y) e^{-\tau y / 4} \int_{0}^{y} \phi(y) e^{\tau y / 4} d z=\phi(y)^{2} .
$$

The last claim follows directly from (23) after the integration of the term $\int_{0}^{y} e^{(\tau-1) z / 4} d z$ giving a sharper estimate for $\tau>1$ but less explicit one. Indeed, this is better estimate as the function $\frac{4}{y(\tau-1)}\left(e^{(\tau-1) y / 4}-1\right)$ can be bounded from below by 1 . It can be ascertained by proving that this function is nondecreasing and has the limit equal to 1 as $y \rightarrow 0^{+}$.

(D) This is a direct conclusion from the estimates (C), monotonicity $\phi(y) e^{y / 4} \searrow$ and the definition of $\mathcal{S}$ in (7). Indeed, one can estimate easily for any $\tau \leq 1$

$$
\mathcal{S} \mathcal{T} \phi(y) \geq e^{-y / 4} \int_{0}^{y} \phi(z)^{2} e^{z / 4} d z \geq e^{-y / 4} \int_{0}^{y} \phi(z) \phi(y) e^{y / 4} d z,
$$

while for any $\tau>1$ one obtains

$$
\mathcal{S} \mathcal{T} \phi(y) \geq e^{-y / 4} \int_{0}^{y} \phi(z)^{2} e^{(2-\tau) z / 4} d z \geq e^{-y / 4} \int_{0}^{y} \phi(z) e^{(1-\tau) z / 4} \phi(y) e^{y / 4} d z .
$$

The last claim in (D), involving lower estimate by the squared mass, follows from

by integration by parts

$$
\mathcal{S T} \phi(y) \geq \phi(y) \int_{0}^{y} \phi(z) d z
$$

$$
\int_{0}^{\infty} \phi(y) \int_{0}^{y} \phi(z) d z d y=\left(\int_{0}^{\infty} \phi(y) d y\right)^{2}-\int_{0}^{\infty} \phi(y) \int_{0}^{y} \phi(z) d z d y .
$$

(E) For $\tau \leq 1$ as before using the monotonicity property $\phi(y) e^{y / 4} \searrow$ we deduce that $\phi(y) \leq|\phi|_{\infty} e^{-y / 4}$ as its maximum value is attained at 0 . Next, we use this inequality to proceed as follows

$$
\mathcal{T} \phi(y) \leq \frac{1}{y} \phi(y)|\phi|_{\infty} e^{-\tau y / 4} \int_{0}^{y} e^{(\tau-1) z / 4} d z
$$

and estimating $e^{(\tau-1) z / 4}$ from above by 1 as $\tau \leq 1$ yields the claim. 
For $\tau \geq 1$ we proceed as for the case $\tau \leq 1$ but this time we estimate $e^{(\tau-1) z / 4}$ from above by $e^{(\tau-1) y / 4}$ for any $z \leq y$. Namely,

$$
\mathcal{T} \phi(y) \leq \frac{1}{y} \phi(y)|\phi|_{\infty} e^{-\tau y / 4} \int_{0}^{y} e^{(\tau-1) z / 4} d z \leq \frac{1}{y} \phi(y)|\phi|_{\infty} e^{-\tau y / 4} \int_{0}^{y} e^{(\tau-1) y / 4} d z .
$$

In this case the monotonicity property $\phi(y) e^{y / 4} \searrow$ was also used to get $\phi(z) \leq|\phi|_{\infty} e^{-z / 4}$.

The last claim follows for any $\tau>0$ and $\int_{0}^{\infty} \phi(z) d z=m$, by $e^{\tau z / 4} \leq e^{\tau y / 4}$ for $z \leq y$, from

$$
\mathcal{T} \phi(y) \leq \frac{1}{y} \phi(y) e^{-\tau y / 4} e^{\tau y / 4} \int_{0}^{y} \phi(z) d z \leq \frac{1}{y} \phi(y) \int_{0}^{y} \phi(z) d z
$$

and in consequence $\mathcal{T} \phi(y) \leq \frac{1}{y} \phi(y) m$.

(F) Due to the monotonicity property $\phi(y) e^{y / 4} \searrow$ the sup norm of the function $\phi(y) e^{y / 4}$ is attained at 0 whence $\phi(y) \leq|\phi|_{\infty} e^{-y / 4}$ follows. Then one applies this estimate to the first property from $(\mathrm{E})$ to derive the required estimate.

$(\mathrm{G})$ This is a direct conclusion from the estimate $(\mathrm{E})$ and the definition of $\mathcal{S}(7)$. For $\tau \leq 1$ we drop the term $e^{(\tau-1) z / 4}$ (cf. the lines of the proof $(\mathrm{H})$ below).

(H) Applying the first estimate from (E) and the inequality $\phi(z) \leq|\phi|_{\infty} e^{-z / 4}$ following from (B) we obtain

$$
\mathcal{S} \mathcal{T} \phi(y) \leq e^{-y / 4} \int_{0}^{y} \mathcal{T} \phi(z) e^{z / 4} d z \leq e^{-y / 4} \int_{0}^{y}|\phi|_{\infty} \phi(z) e^{1-\min \{\tau, 1\} z / 4} d z .
$$

Integration of the last term gives the required estimate.

(I) First by $\phi(y) e^{y / 4} \searrow$ we have $\phi(y) \leq e^{-y / 4} \sup _{z \geq 0} \phi(z) e^{z / 4}$. Then integration yields $m=\int_{0}^{\infty} \phi(y) d y \leq 4 \sup _{z \geq 0} \phi(z) e^{z / 4}$. The next lower bound follows by (B) and the lower estimates for $\mathcal{S T}$ (D) from

$$
\sup _{y \geq 0} \mathcal{P} \phi(y) e^{y / 4}=\mathcal{P} \phi(0)=\frac{m}{4}+\frac{1}{8} \int_{0}^{\infty} \mathcal{S} \mathcal{T} \phi(y) d y \geq \frac{m}{4}+\frac{m^{2}}{16} .
$$

(J) The property follows from straightforward calculations after substitution of the function $\phi(y)=\frac{1}{4} m e^{-y / 4}$ in the definition of the operator $\mathcal{P}$.

Acknowledgements. The preparation of this paper was partially supported by the Polish Ministry of Science Project N 201022 32/0902 and by the European Commission Marie Curie Host Fellowship for the Transfer of Knowledge "Harmonic Analysis, Nonlinear Analysis and Probability" MTKD-CT-2004-013389.

\section{References}

[1] P. Biler, Growth and accretion of mass in an astrophysical model, Appl. Math. (Warsaw) 23 (1995), 179-189.

[2] P. Biler, Local and global solvability of some parabolic systems modeling chemotaxis, Adv. Math. Sci. Appl. 8 (1998), 715-743.

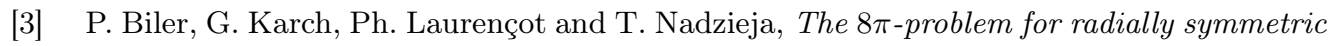
solutions of a chemotaxis model in the plane, Math. Methods Appl. Sciences 29 (2006), 1563-1583.

[4] P. Biler, A note on the paper of Y. Naito, in: Banach Center Publ. 74 (2006), 33-40. 
[5] A. Blanchet, J. Dolbeault and B. Perthame, Two dimensional Keller-Segel model: Optimal critical mass and qualitative properties of the solutions, Electron. J. Diff. Eqns. 44 (2006), $1-33$.

[6] S. Luckhaus and Y. Sugiyama, Large time behavior of solutions in subcritical cases to degenerate Keller-Segel systems, Mathematical Modelling and Numerical Analysis 40 (2006), 597-621.

[7] Y. Naito, Non-uniqueness of solutions to the Cauchy problem for semilinear heat equations with singular initial data, Math. Ann. 329 (2004), 161-196.

[8] Y. Naito, Asymptotically self-similar solutions for the parabolic systems modelling chemotaxis, in: Banach Center Publ. 74 (2006), 149-160.

[9] Y. Naito and T. Suzuki, Self-similarity in chemotaxis systems, Colloq. Math. 111 (2008), $11-34$.

[10] Y. Naito, T. Suzuki and K. Yoshida, Self-similar solutions to a parabolic system modeling chemotaxis, J. Differential Equations 184 (2002), 386-421.

[11] R. Stańczy, Hammerstein equations with an integral over a noncompact domain, Ann. Polon. Math. 69 (1998), 49-60.

[12] Y. Sugiyama, Global existence and finite time blow up for some degenerate Keller-Segel model, in: Variational Problems and Related Topics, Y. Naito and T. Suzuki (eds.), Sūrikaisekikenkyūsho Kōkyūroku 1464, Kyoto, 2005, 40-57.

[13] Y. Sugiyama and H. Kunii, Global existence and decay properties for a degenerate KellerSegel model with a power factor in drift term, J. Differential Equations 227 (2006), 333364 . 